\title{
SUR UN TRICHOMONADIDÉ LIBRE DES EAUX STAGNANTES
}

\author{
Par G. LA VIER
}

On connaît actuellement un nombre considérable d'espèces appartenant aux genres Trichomonas ou Eutrichomastix et jusqu'à l'année dernière on pouvait penser que les Trichomonadidés étaient exclusivement des parasites se rencontrant dans le tube digestif, secondairement parfois dans le sang, d'hôtes très variés. A. Bishop a relaté récemment (1935) l'existence dans l'eau d'une mare du Lincolnshire (Angleterre) d'un flagellé à vie libre pour lequel elle a a créé l'espèce « Trichomonas » keilini (les guillemets du nom générique exprimant son doute quant à la certitude d'attribution); elle en a obtenu la culture sur divers milieux et en a décrit la morphologie et le mode de division.

Au cours de recherches sur les Diplomastigines libres de l'eau, il m'a été donné de rencontrer un Trichomonadidé que je crois pouvoir assimiler à celui d'A. Bishop, dans deux échantillons différents d'eau stagnante :

Eau $n^{\circ} 191$ : Echantillon prélevé le 24 novembre 1935 dans une mare située sur la commune de Fresnes (Seine), à proximité de la Croix-deBerny, et provenant d'infiltrations de la rivière Bièvre ; volume : $250 \mathrm{cc}$. environ, contenant des végétaux, des mollusques, des larves d'Anopheles bifurcatus, des Hydracariens, des Copépodes et Ostracodes, une dizaine d'espèces d'Infusoires; au point de vue des Flagellés: Trepomonas très abondants ( $T$. agilis, $T$. angulata, $T$. rotans), nombreux Hexamita, Tetramitus descissus et $T$. piriformis, etc.; peut-être à ce moment une trichomonade a-t-elle été vue à l'examen direct ? En tout cas, le 12 décembre, elles sont nombreuses et le restent jusqu'au 2 janvier 1936 ; leur nombre décroît ensuite ; le 6 janvier, ils sont devenus rares; ce qui va dominer à partir de ce moment, c'est Trepomonas rotans ( $T$. angulata et $T$. agilis ayant disparu après une quinzaine de jours) ; cependant l'ensemencement de trois à quatre gouttes d'eau permet encore d'obtenir chaque fois une poussée de trichomonades. L'échantillon d'eau qui avait été à deux reprises additionné d'eau stérile est abandonné à la dessiccation. Le 5 mars, il ne reste plus dans le fond du flacon qu'un dépôt pulvérulent et sec ; le 20 mars, 200 cc. d'eau stérile sont versés dans le flacon, nous verrons plus loin avec quel résultat.

Annales de Parasitologie, T. XIV, N $^{\circ} 4 .-1^{\text {er }}$ juillet 1936, p. $359-368$. 
A partir de cet échantillon 191, des cultures primaires impures ont été obtenues le 4 décembre, le 8 , le 18 , le 22 , le 13 janvier, le 24 février et encore après l'addition d'eau sur le dépôt sec. Des subcultures ont été faites et continuent encore actuellement.

Eau $n^{\circ} 201$ : Echantillon de 100 cc. environ, prélevé le $1^{\text {er }}$ janvier 1936 dans un fossé à eau permanente situé à Roncevaux, en bordure des marécages de Malesherbes (Loiret) alimentés par l'Essonne; contenant quelques mollusques (Planorbes), des Copépodes et Ostracodes très abondants ; infusoires nombreux d'espèces variées parmi lesquels un Gyrocoris qui malheureusement avait disparu après quelques heures. Pour les Flagellés : Trepomonas surtout ( $T$. angulata dominant) ; Tetramitus descissus et $T$. piriformis qui ont déjà disparu le 3 janvier ; à cette dernière date des Trichomonades sont vues; dès le 2, des ensemencements avaient été faits qui donnèrent une culture abondante : cette souche existe encore en subcultures.

Culture. - A. Bishop a essayé avec de bons résultats un certain nombre de milieux de culture de nature diverse. Je n'ai pas recherché un optimum cultural mais seulement un milieu facile pour la conservation de la souche; pour cela je m'en suis bientôt tenu au liquide ovomucôïde de Boeck, non pas que ce milieu fournisse une culture extrêmement abondante mais parce qu'il est peu favorable à la pullulation bactérienne ; il permet ainsi dans les cultures impures d'assurer une meilleure conservation des trichomonades ; celle-ci est encore améliorée par l'addition d'une couche d'huile de paraffine. On sait de longue date déjà que les besoins en oxygène des Trichomonas sont faibles sinon nuls et cela a déjà été utilisé pour leur culture ; une couche d'huile n'assure à vrai dire qu'une anaérobiose relative ; mais celle-ci est néanmoins suffisante pour éliminer certains microorganismes nuisibles, en particulier les Polytoma qui, lorsqu'ils sont présents, se développent avec une exubérance telle que les autres Flagellés disparaissent rapidement; peut-être aussi cela favorise-t-il la pousse de bactéries fournissant pour les trichomonades une nourriture meilleure, ou empêche-t-il au contraire celle d'organismes nuisibles. Le fait est que chaque fois que j'ai simultanément ensemencé deux tubes de liquide ovomucoïde l'un avec couche d'huile, l'autre avec surface libre, les trichomonades avaient disparu du second après 5 semaines en moyenne, alors qu'il en existait encore, de rares il est vrai, dans les premiers après plus de 4 mois. Par ce procédé, un repiquage tous les trois mois est donc suffisant. Cette anaérobiose ne suffit pas à éliminer d'autres Flagellés qui accompagnent normalement les trichomonades et ont des besoins similaires comme les Trepomonas et les Hexamita; mais ceux-ci, dans le liquide ovomucoïde, 
se développent moins abondamment que les trichomonades et ne paraissent pas leur nuire.

Inutile d'ajouter que le milieu de culture est laissé à la température ordinaire du laboratoire ; j'ai pu vérifier le fait déjà noté par A. Bishop, que la température de $37^{\circ}$ est léthale.

Morphologie du Flagellé vivant. - Soit dans l'eau, soit dans les cultures, ce qui attire l'attention sur le Flagellé c'est son mode de progression bien différent de celui des autres formes libres; il se dirige suivant une ligne sensiblement droite et pour ce faire se courbe de façon saccadée alternativement de chaque côté ; ce mouvement d'oscillation n'intéresse que la partie antérieure, plus large, du corps, l'extrémité postérieure suivant toujours le même mouvement de translation. L'animal avance ainsi trop rapidement pour que l'on puisse bien voir sa structure, mais très fréquemment il lui arrive, comme à presque tous les Flagellés, de trainer à son extrémité postérieure des débris adhérents qui freinent le mouvement et permettent ainsi un meilleur examen.

Il se présente sous les deux aspects principaux signalés par A. Bishop : globuleux ou allongé ; entre les deux existent d'ailleurs toutes les formes de passage. La longueur varie de 6 à $12 \mu$; la largeur de 3 à 7 . Les formes globuleuses montrent toujours un éperon axostylaire bien net, fin et très aigu ; les formes les plus allongées montrent un éperon très court ou même rien ne dépassant à l'extrémité ; l'axostyle dans ce cas n'en est certainement pas moins présent, mais est invisible, le cytoplasme est d'ailleurs capable, comme nous le verrons plus loin, de déplacer dans de larges limites son pòle inférieur le long de l'axostyle, de sorte que la partie saillante de celui-ci est variable ; assez souvent aussi il est recouvert en partie par une sorte de bourrelet cytoplasmique (fig. 1,3 ; fig. 3,3 ). L'axostyle ne peut être suivi à l'intérieur que pendant un très court trajet. Le cytoplasme est finement vacuolaire, souvent bourré d'inclusions alimentaires. On ne distingue pas le noyau ; un cytostome est assez souvent visible sous forme d'une petite encoche latérale vers l'extrémité antérieure.

Les flagelles libres sont au nombre de trois, comme on peut s'en assurer si l'on tue le protozoaire entre lame et lamelle en laissant fuser un peu de liquide de Lugol ou une solution de quinine. Dans la vie, ils présentent un mouvement en vortex réalisé en deux temps dont le deuxième est brusque, à la manière du cocher qui fait claquer son fouet ; c'est ce mouvement qui produit la progression saccadée. De leur point d'insertion part en arrière une membrane on- 


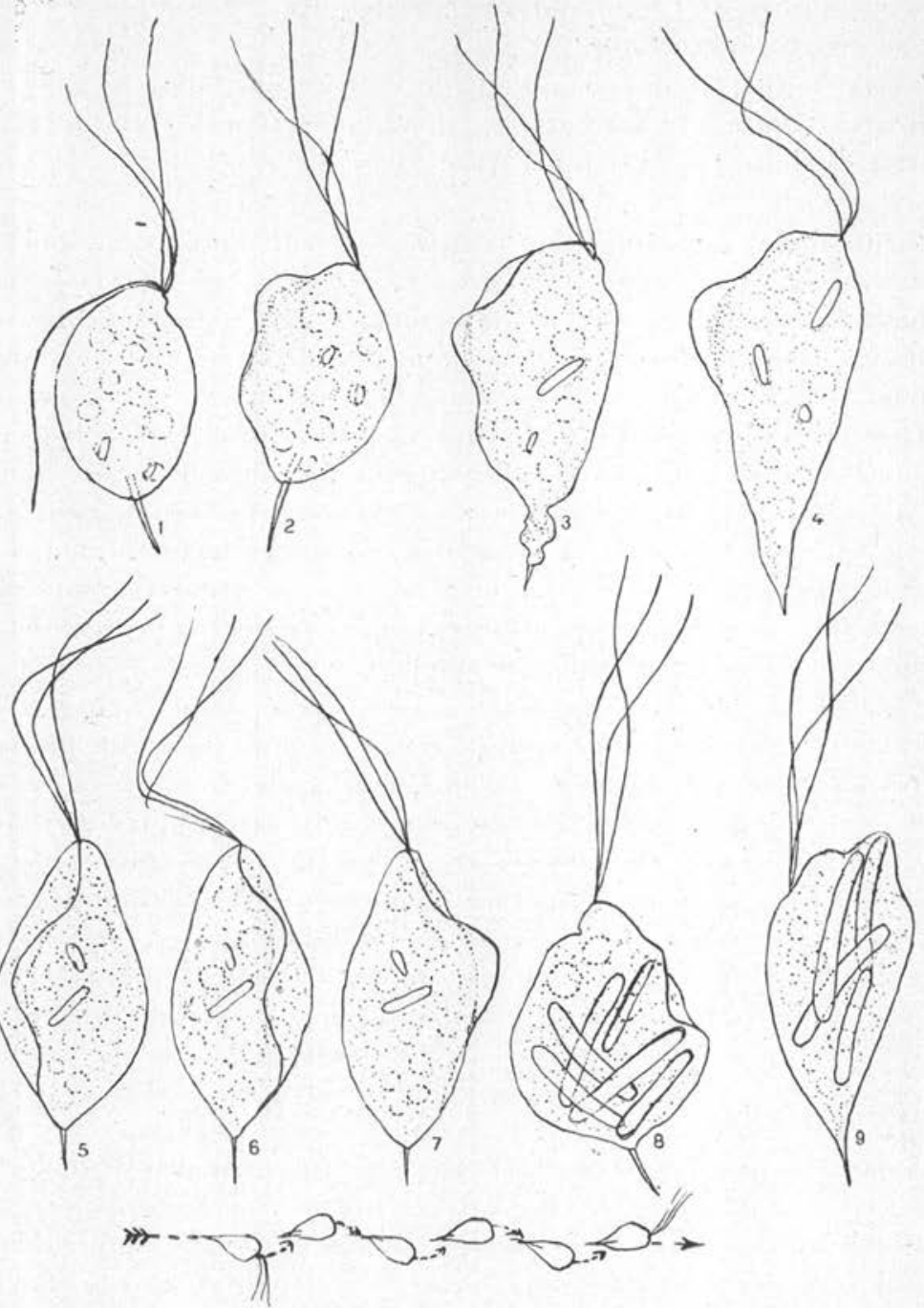

FıG. 1. - Eutrichomastix keilini de l'eau stagnante, d'après des croquis sur le vivant: 1 , forme avec flagelle récurrent entièrement libre; 2 , forme globuleuse ; 3-4, formes allongées ; 5-7, trois aspects' successifs du même individu pour montrer les mouvements des flagelles libres et la rotation du flagelle adhérent ; 8-9, individus bourrés de bactéries. En bas, représentation schématique du mode de progression.

dulante; celle-ci est déterminée par un quatrième flagelle; les ondulations sont grossières ; il n'y en a qu'une ou deux vagues à la 
fois. Le mouvement de ce quatrième flagelle est assez curieux : en même temps que l'onde progresse d'avant en arrière le flagelle glisse en tournant sur la surface du corps (fig. 1, 5-7) ; cette rotation rappelle bien le mouvement du flagelle récurrent des Eutrichomastix. Le flagelle adhérent ne dépasse pas en longueur le pôle postérieur, souvent même il ne l'atteint pas ; son adhérence est variable; elle est généralement forte, mais, contrairement à A. Bishop, j'ai vu souvent ce flagelle se libérer à son extrémité en étirant une mince languette cytoplasmique (fig. 2, 1-4); à plusieurs reprises, je l'ai même vu entièrement libre (fig. 1, 1) et on a alors une forme Eutrichomastix typique.

Tels sont les aspects que l'on rencontre chez les individus qui nagent librement; souvent des Flagellés sont empêchés de progresser par l'adhérence soit à la lame ou la lamelle, soit à de gros débris ou des amas bactériens ; ils sont dans ce cas susceptibles de présenter une métabolie intense; sur ceux dont l'axostyle est ainsi " ancré » par adhérence, ce qui est un cas très fréquent, j'ai observé un curieux mouvement vigoureux et saccadé du cytoplasme d'avant en arrière et réciproquement le long de la tige axostylaire immobilisée (fig. 2, 5-9) ; les efforts sont si intenses que l'axostyle disparait entièrement, comme avalé, et qu'entre le petit renflement céphalique et la sphère postérieure formée par la condensation du cytoplasme il ne reste qu'un étroit pont tubulaire pour recouvrir l'axostyle. Malgré la violence du mouvement qui peut se manifester pendant fort longtemps (je l'ai suivi sur le même individu pendant plus de 20 minutes), il ne se produit pas de rupture. Quand la mort survient, le Flagellé se met en boule, la pointe axostylaire disparait à l'intérieur et les flagelles qui battaient encore faiblement s'immobilisent.

Le vert janus colore vitalement une assez grosse granulation antérieure qui est vraisemblablement le corps parabasal et de petites granulations périphériques, sans doute des mitochondries.

Ce Flagellé est très vorace ; il est capable d'ingérer des proies dont la taille est relativement énorme pour lui ; certains individus en sont littéralement bourrés, surtout d'un grand bacille dont ils paraissent particulièrement friands ; parfois ils arrivent à être complètement déformés par leur contenu (fig. 1, 8-9).

J'ai pu suivre sur le vivant une partie des phénomènes de division, celle qui a trait à la cytodiérèse (fig. 2). Lorsque je rencontrai le Flagellé représenté, la mitose était certainement terminée puisqu'il y avait déjà deux membranes ondulantes et que les nouveaux flagelles avaient poussé ; l'enśemble avait une forme ovalaire qui 


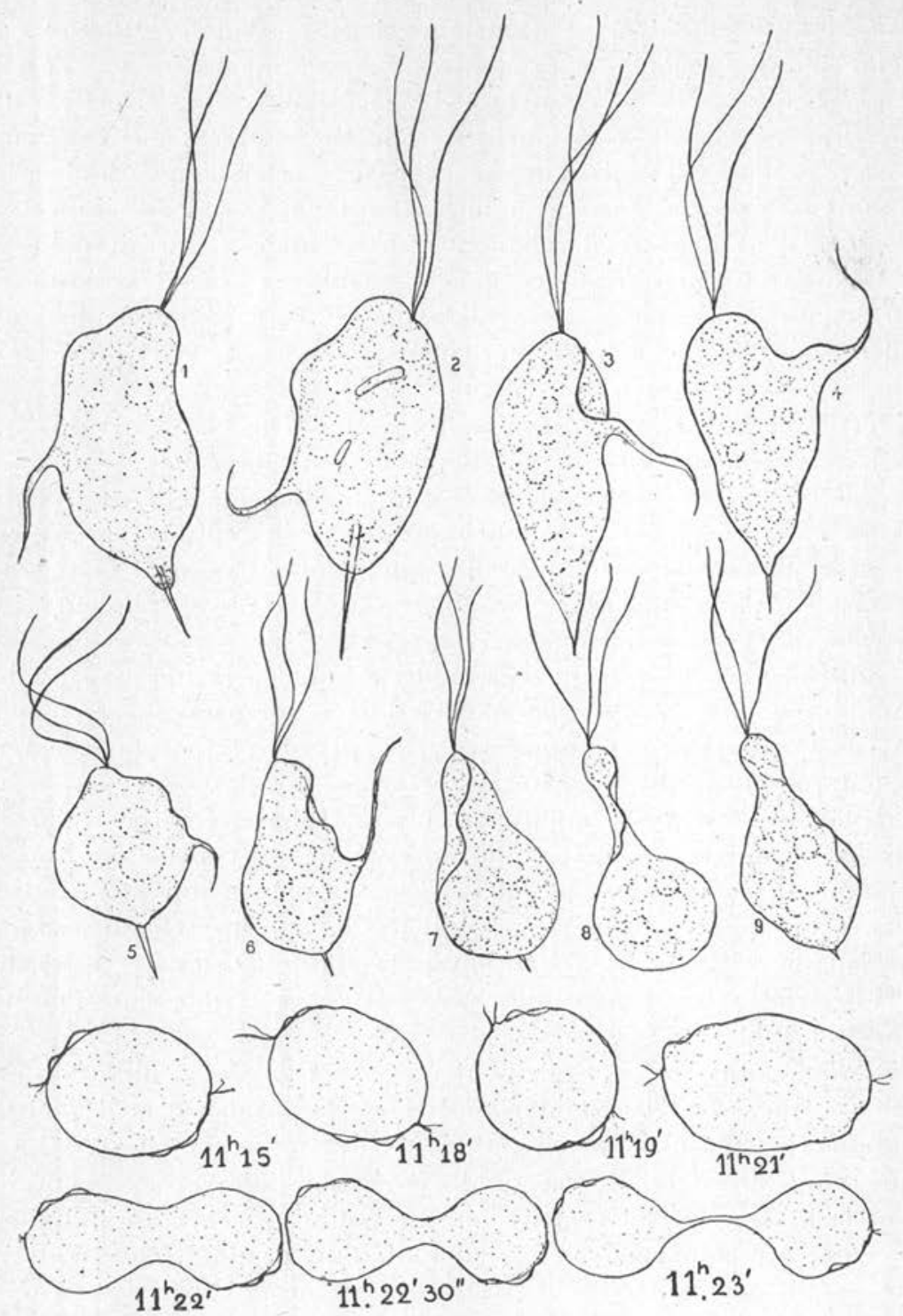

Frf, 2. - Eutrichomastix keilini de l'eau stagnante, d'après des croquis sur le vivant: 1-4, formes avec flagelle récurrent partiellement adhérent ; 5-9, aspects successifs d'un même individu « ancré » par sa pointe axostylaire, montrant le mouvement de translation en masse du cytoplasme le long de l'axostyle. En bas : plasmodiérèse. 
persista plusieurs minutes sans changement, simplement avec un lent mouvement de rotation sur place; brusquement se produisit une condensation en sphère, la rotation cessa, la forme redevint ovalaire et le grand axe commença immédiatement à s'allonger ; l'étirement dura une minute, puis une encoche apparut de chaque côté de la région médiane et une minute encore plus tard les deux individus étaient constitués, reliés seulement par un mince pont protoplasmique qui cassa trois minutes plus tard (à 11 h. 26' ; non représenté sur la figure). Cette rapidité de la plasmodiérèse est assez rare chez les Flagellés.

Morphologie du Flagellé coloré. - La fixation (liquide de Bouin) et la coloration (hématoxyline ferrique) permettent de mettre en évidence la structure (fig. 3) ; mais, comme déjà A. Bishop, j'ai éprouvé qu'il n'était pas facile d'obtenir de bonnes préparations.

L'axostyle apparaît comme une mince baguette sidérophile, mais dont la chromatophilie est bien moins marquée que celle des flagelles; il n'est pas toujours possible de le suivre entièrement ni même de le voir ; mais je ne pense pas que l'on soit dans ce dernier cas autorisé à parler de l'absence de cet organite, car chez beaucoup de flagellés analogues, d'une fixation beaucoup plus aisée, on rencontre toujours des individus qui, moins bien fixés ou moins bien colorés, ne le laissent pas voir. Le noyau ovalaire ou sphérö̈de mesure en moyenne de 1,5 à $2 \mu$ suivant son grand axe. Il présente un assez volumineux karyosome subcentral. Le cytoplasme est vacuolaire, plus dense dans la région nucléaire et un individu (fig. 3,2 ) m'a montré nettement, dans cette région, les granulations sidérophiles paranucléaires qui sont si fréquentes chez les Trichomonas et les Eutrichomastix. A l'extrémité antérieure, le blépharoplaste volumineux m'a toujours paru unique quoique non exactement sphérique ; il est possible qu'à son voisinage immédiat soit situé un parabasal correspondant à la granulation colorable vitalement par le vert janus ; les fixations osmiques (Champy, Flemming) ne m'ont toutefois pas donné un aspect différent de celui produit par le Bouin acétique ; je n'ai, il est vrai, obtenu avec elles que des préparations trop peu claires pour être affirmatif à ce sujet. Il n'y a pas de costa (fibre basaie). Du blépharoplaste partent les quatre flagelles : les trois antérieurs, libres, sont inégaux, la différence entre chacun étant de l'ordre de quelques $\mu$ (fig. 3,5 ). Le flagelle récurrent court le long du corps en lui adhérant. Je n'ai pas observé dans les préparations de terminaison libre de ce flagelle, mais le nombre des individus que j'ai ainsi 
examinés est infiniment plus restreint que celui des exemplaires vivants. Je n'y ai pas trouvé non plus de formes en division ; celle-ci a été décrite en détail par A. Bishop.

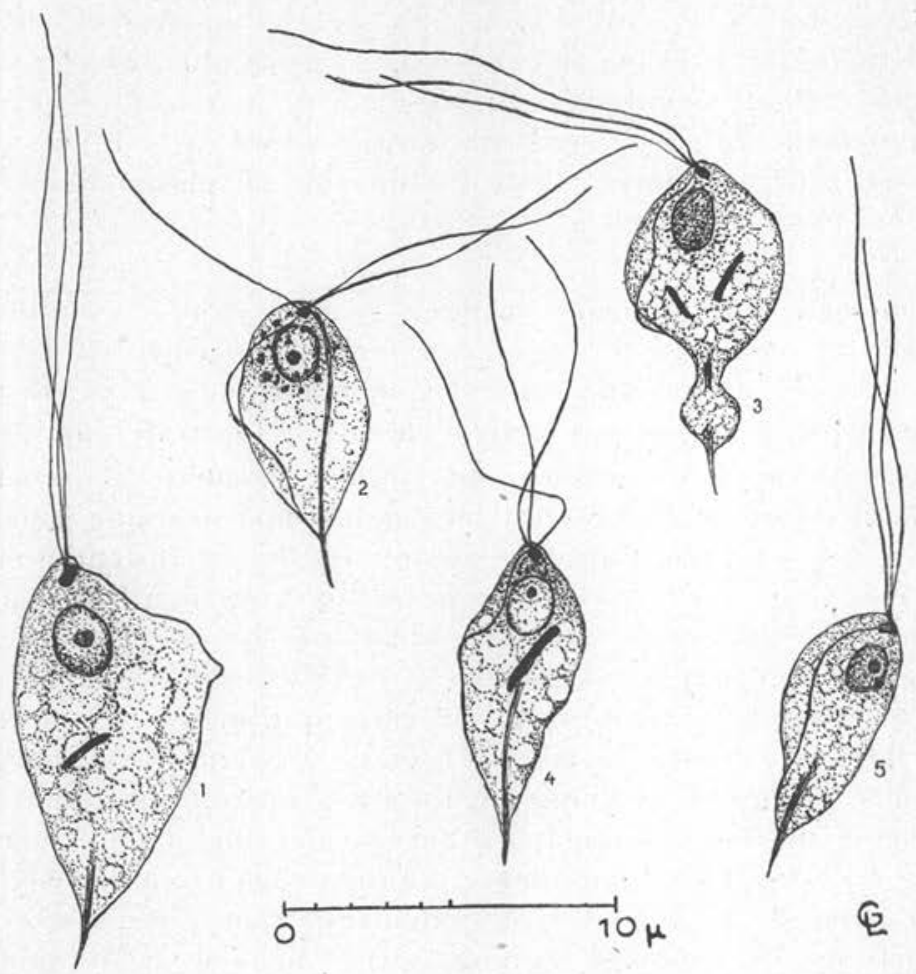

Fıc. 3. - Eutrichomastix keilini de l'eau stagnante (Bouin ; hématoxyline ferrique) : 1, grand individu; 2, petit individu particulièrement bien fixé montrant l'axostyle en entier, la structure nucléaire et les granulations paranucléaires ; $3-4$, formes courantes ; 5 , individu montrant bien la différence de taille des flagelles.

Formes de résistance. - Le Flagellé de l'eau possède certainement une forme de résistance, comme le prouve l'expérience suivante : l'échantillon 191 ayant été, comme je l'ai relaté plus haut, abandonné à la dessiccation, le dépòt pulvérulent est abandonné pendant quinze jours, et le 20 mars on ajoute environ $100 \mathrm{cc}$. d'eau de robinet bouillie ; quelques heures après, des Infusoires ont reparu et augmentent en nombre et en espèces les jours suivants (Colpodes, Paramécies, Cyclidium); le 25 mars, en plus quelques Tetramitus et 
un Bodo ; le 29 , une trichomonade est vue ; le $1^{\text {er }}$ avril, le nombre en a sensiblement augmenté ; le 28 avril, elles ont presque disparu ; au 10 mai, il n'en est plus vu; par contre, Treponomas rotans a fait son apparition et augmente ensuite peu à peu en quantité. A deux reprises, la trichomonade a été ensemencée avec succès en milieu ovomucoïde et les subcultures sont continuées.

Il n'y a donc pas de doute que notre Flagellé de l'eau ne puisse résister à une dessiccation totale de deux semaines. Sous quelle forme vit-il alors ? c'est ce que je ne puis dire actuellement ; l'examen microscopique du dépôt pulvérulent m’a montré une extraordinaire abondance de formes kystiques de toutes tailles et défiant toute identification.

Les deux souches que j'ai isolées se sont montrées tout à fait similaires. Les dimensions du Flagellé-sont les mêmes qu'a données A. Bishop ; la morphologie aussi est identique, à la seule différence près que j'ai observé des formes à flagelle récurrent partiellement ou totalement libre. Il n'y a donc pas de doute que j'ai eu affaire à la même espèce. Mais il reste à trancher un point, celui du nom générique qu'elle doit porter. A. Bishop a bien montré que celui de Trichomonas ne la satisfaisait pas et il n'y a pas de doute en effet qu'il ne puisse être correctement appliqué ici. Doflein le premier, puis après lui nombre d'auteurs ont soutenu que les noms Trichomonas et Trichomastix (devenu plus tard Eutrichomastix) désignaient non des genres différents mais des formes seulement des mêmes Flagellés. Depuis que l'on a fait de nombreuses cultures en souches pures, on a pu constater que la transformation d'une forme en l'autre ne se produisait en réalité jamais, et cette théorie n'est . plus soutenable. Que l'on veuille voir en Eutrichomastix un genre différent de Trichomonas ou seulement un sous-genre de celui-ci, il y a entre les deux une barrière que précise d'ailleurs la morphologie : dans le genre Trichomonas sensu stricto la membrane ondulante est toujours tendue entre le flagelle ondulant et la costa (fibre basale). C'est donc la présence ou l'absence de celle-ci qui fera la démarcation, car on sait depuís longtemps que le flagelle récurrent d'un Eutrichomastix vrai est capable d'adhérer au corps et de déterminer ainsi une membrane ondulante sous l'influence d'une variation soit de viscosité (Chatton, 1920), soit de $p \mathrm{H}$ (Grassé, 1926). Cette adhérence est plus ou moins parfaite, elle peut être totale ou partielle et Grassé a obtenu expérimentalement avec Eutrichomastix colubrorum des formes rappelant singulièrement celles que 
j'ai observées dans le Flagellé de l'eau. Mais la membrane ondulante ainsi obtenue n'est pas en réalité comparable à celle d'un Trichomonas véritable puisqu'elle n'est pas sous-tendue par une costa ; le Flagellé n'est pas devenu un Trichomonas, il est resté un Eutrichomastix. Cela, et au surplus la présence, rare, il est vrai, mais sûre de formes à flagelle récurrent complètement libre montre que le flagellé de l'eau doit se nommer Eutrichomastix keilini Bishop.

Son cas, à ce point de vue, n'est d'ailleurs pas isolé. Récemment, Das Gupta (1936) a décrit la morphologie d'un «Trichomonas 》 du boa constrictor dans des cultures obtenues par Reichenow et d'un «Trichomonas 》 sp. du serpent américain Dymarchon corais; chez tous deux il note l'absence totale de fibre basale et la possibilité pour le flagelle ondulant de se détacher. Pour ces Flagellés de Reptiles, le problème se pose donc exactement de la même façon que pour celui de l'eau et la conclusion doit être la même : tous deux doivent rentrer dans le genre Eutrichomastix.

\section{BIBLIOGRAPHIE}

Bishop (A.). - The morphology and method of division of Trichomonas. Parasitology, XXIII, 1931, p. 129.

- Observations upon a «Trichomonas » from pond water. Ibid., XXVII, 1935 , p. 246.

Chatron (E.). - Culture indéfinie d'un Trichomonas intestinal du cobaye; essais de purification; action morphogène des milieux sur la membrane ondulante. C.R. Soc. de Biol., LXXXIII, 1920, p. 69.

DAS Gupta (B. M.). - Observations on the Flagellates of the genera Trichomonas and Eutrichomastix. Parasitology, XXVIII, 1936, p. 195.

- Trichomonas sp. from the gut contents of a corais snake. Ibid., p. 202. Grussé (P. P.). - Contribution à l'étude des Flagellés parasites. Arch. Zool. expérim. et gén., LXV, 1926 , p. 345 .

Laboratoire de Zoologie et Parasitologie de la Faculté de médecine de Lille.

(Directeur: Prof. G. Lavier). 